\title{
Interference Mitigation Technique through Shielding and Antenna Discrimination
}

\author{
Lway Faisal Abdulrazak ${ }^{1}$ and Arshed A. $\mathrm{O}^{2}$ \\ ${ }^{I}$ Department of Computer Science, Cihan University, Sulaimanyia, Iraq \\ ${ }^{2}$ Faculty of Manufacturing Engineering, UMP, Malaysia ${ }^{2}$ \\ ${ }^{1}$ lway@cihanuniversity-sul.org, ${ }^{2}$ techguy@gmail.com
}

\begin{abstract}
Interference mitigation between IMT-Advanced and Fixed Satellite Services (FSS) is presented in this article. Toward this goal, an analytical model has been developed based on the deterministic analysis of the propagation model. The IMT-Advanced system parameters have been represented by Worldwide Interoperability for Microwave Access (WiMAX) 802.16e. The use of VSAT unit and different shielding materials is considered. The testing is performed in the Anechoic Chamber as well as outdoor, and deployment is designed to fulfill FSS signal receiving criteria. A strong positive correlation was found between path loss parameters various terrains for different deployment areas. The Antenna discrimination has been discussed alongside the shielding absorption coefficients of the suggested materials. Then the antenna discrimination proposal is demonstrated with a high degree of capability to reduce the harmful power interference from IMT-Advanced base station towards the FSS receiver.
\end{abstract}

Keywords: IMT-Advanced, interference, mitigation, satellite, shielding, separation distance, guard band

\section{Introduction}

Wireless This paper addresses the (3400-4200) MHz band of the spectrum, which has been proposed by the International Telecommunication Union of Research (ITU-R) as the widest band that will be available, up to $100 \mathrm{MHz} / \mathrm{channel}$, for the future International Mobile Telecommunication Advanced (IMT-Advanced) operational frequency. For FSS, C-Band is used in many countries, represented by thousands of strategic investments ranging from Telemedicine and distant learning to disaster recovery [1]. Accordingly, any immediate transition in the use of this band to IMT-Advance services is considered unrealistic [2]. The super extended C-band 3400-4200 MHz is attractive for FSS because of its low absorption, highly reliable space to earth communication and wide service coverage. In addition, this frequency band is widely used by satellite operators in the countries with severe rain fade conditions due to almost zero rain-induced signal attenuation. C-band is also favorable to IMT-Advanced, because it allows multiple antenna technique implementations, and the use of smaller antenna for terminals and base stations; as well as enabling high space efficiency [3].

The co-channel interference $(\mathrm{CCI})$ and adjacent channel interference $(\mathrm{ACI})$ are issues that result of co-locating more than one service in one band. However, CCI is the worst of the issues in the co-existence of both IMT-Advanced and FSS using the same frequency carrier. ACI results from other signals that are adjacent in the frequency to the desired signal.

The sharing results by using a Minimum Coupling Loss (MCL) and Monte-Carlo (MC) simulation link gave a required separation distance larger than $40 \mathrm{~km}$ to avoid mutually 
harmful interference between two systems in co-channel and adjacent channel interference scenario $[4,5]$. On the IMT-Advanced side, the Orthogonal Frequency Division Multiplexing (OFDM) is currently considered the most promising access schemes to support IMTAdvanced systems [6]. It is based on multi-carrier modulation technique that offers excellent performance in combating multi-path fading as well as superb efficiency in terms of using the available bandwidth [7].

Shielding in this paper is used to attenuate the Electromagnetic Incompatibility (EMI) between sources (IMT-Advanced) and susceptible equipment (FSS receiver). The mechanism of shielding is described as follows: when terrestrial waves hit the shield, a part of its energy will be reflected because of the shield surface; another part of the energy will be absorbed and transformed to other shapes of energy (thermal and electrical energy). Part of the electrical energy will be discharged through the ground and the rest will pass through the shielding. So, basically the site shielding is about physical obstruction built to reduce the interference from the interferer to the victim receiver [8].

Most of the studies recommended that shielding can reduce the harmful interference [9]. The best isolation happened when the enclosure is fabricated as one homogeneous piece. The shielding material choice is wide, but each material differs in its ability to attenuate the electromagnetic waves. Shielding can be natural by locating the FSS dish in around the back of building or hill. It can be done artificially by adding one or two walls on the path between victim and interferer [8]. Two walls will be much better because it will duplicate the amount of attenuation. The dish elevation angle should be considered during shielding deployments to prevent signal blocking by the shielding shape. By putting the dish as low as possible, and with high shielding all around except in the direction of the beam to satellite, will help to avoid the interference. The signal reception will be better, because the more the dish is hidden the greater the reduction in interference level [10].

A new practical shielding mitigation technique is needed to achieve the minimum separation distance. This technique can further increase the possibility of sharing between these systems using guard band insertion between the two services. The shielding strategy developed base on test bed measurements to evaluate the attenuation of the proposed materials. Matlab ${ }^{\mathrm{TM}}$ has been used as a simulation tool, whereas the IMT-Advanced parameters have been characterized by WiMAX IEEE802.16e. The impact of different FSS channel bandwidths, guard band separations, shielding effects, antenna heights and different deployment areas on co-existence feasibility are considered.

\section{Propagation Model and Systems Parameters}

The shielding technique $(\mathrm{R})$ can attenuate the interference power, where $\mathrm{R}$ may take a value between $0 \mathrm{~dB}$ to $40 \mathrm{~dB}$ depending on the materials and shielding arrangement, as clarified below [11]:

$$
\begin{aligned}
& 20 \log (d)=-I+\text { EIRP }_{\text {Interferer }}-92.5 \\
& -20 \log (F)-A_{h}+G_{v s}(\alpha)-R
\end{aligned}
$$

Where $A_{h}$ is the factor related to the territories as described earlier in this chapter, $d$ is separation distance, $R$ is the shielding loss, EIRP: is the effective isotropic radiated power transmitted from the interferer, $\mathrm{F}$ is the frequency and $G_{v s}$ is related to the typical receiving FSS antenna gain [12], [13].

Intuitively by introducing clutters, smaller separation distance is achieved and vice versa. Path loss prediction in the case of Line Of Sight (LOS) is obtained by including the losses 
produced by the line-of-sight situation together with the losses produced by clutter models as shown in Equation (2) [4]:

$$
\begin{aligned}
& L(d)=92.44+20 \log _{10} f_{G H z}+20 \log _{10} d_{K m} \\
& \left.+10.25 e^{-d_{k}} \mid 1-\tanh \left[6\left(\frac{h}{h_{a}}-0.625\right)\right\rceil\right] \mid-0.33
\end{aligned}
$$

Where $d$ is the distance between the interferer and the victim receiver in kilometers, $f$ is the carrier frequency in Gega Hertz and $d_{k}$ is the distance in $\mathrm{km}$ from nominal clutter point to the antenna $(\mathrm{dk}=0.02 \mathrm{~km}, 0.02 \mathrm{~km}, 0.025 \mathrm{~km}$ and $0.1 \mathrm{~km}$ for the four deployment environments dense urban, urban, sub-urban and rural, respectively), $h$ is the antenna height (m) above local ground level and ha is the nominal clutter height above local ground level (ha= $25 \mathrm{~m}, 20$ $\mathrm{m}, 9 \mathrm{~m}$ and $5 \mathrm{~m}$ for the four deployment environments).

The receiving gain of FSS station is called off axis antenna $G_{v s}(\alpha)$. The off axis angle value depends on the earth station location and the main receiving beam, where a typical receiving antenna gain can be calculated as Equation (3) [4]:

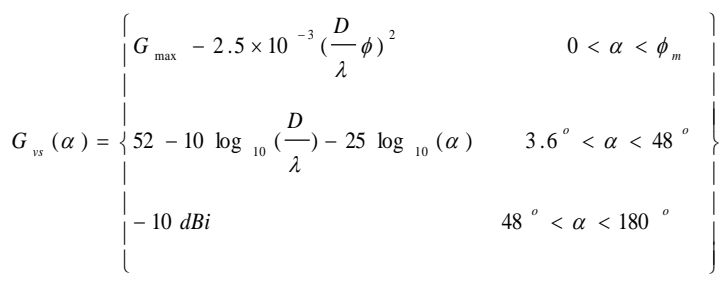

Where Gmax is the maximum antenna gain (38dBi), $\mathrm{D}=1.8 \mathrm{~m}$ (satellite diameter) and $\lambda$ is the wave length in meter and $\phi_{m}$ is given by:

$$
\phi_{m}=\frac{20 \lambda}{D} \sqrt{G_{\max }-2-15 \log _{10}\left(\frac{D}{\lambda}\right)}
$$

In the simulation a value of $-10 \mathrm{~dB}$ was considered to represent the local case study (the FSS elevation angle at the experiment location was $74^{\circ}$ ).

In addition to the deterministic approach, other critical parameters, such as the receiver blocking and Adjacent Channel Interference Ratio (ACIR) are considered in this work. These resulted from the introduction of the Spectrum Emission Mask (SEM) of the interferer and the blocking filter capability of the victim. It is worth mentioning that receiver blocking and ACIR calculations are based on transmitter SEM and victim filter response powers [14]. The receiver blocking is considered in order to find the power degradation in decibel. This can be calculated as follows:

$$
\text { Receiver Blocking }=\left\{\begin{array}{lll}
A C S & \text { Re duction } & \text { if } B W_{\text {int efferer }}<B W_{\text {victim }} \\
0 d B & \text { if } B W_{\text {int efferer }} \geq B W_{\text {victim }}
\end{array}\right\}
$$

Such a SEM is the $20 \mathrm{MHz}$ channel bandwidth type-G WiMAX spectrum emission mask in [15]. In order to calculate the adjacent channel interference, the ACIR should be considered by reducing the interference powers of the interferer ACLR and the victim ACS which are located on different central frequencies [16]. The ACIR is given by: 


$$
A C I R=10 \log 10\left[\left(10{ }^{A C L R}{ }_{I M T} / 10\right)^{-1}+\left(1{ }^{A C S}{ }_{F S S} / 10\right)^{-1}\right]^{-1}
$$

With the carrier frequency at $4 \mathrm{GHz}$, the overall propagation model may be rewritten as follows:

$$
\begin{aligned}
& 20 \log _{10}(d)=-I+\text { EIRP }_{\text {wiMAx }}+G_{v s}(\alpha)-104.58+\text { ACIR } \\
& + \text { corr_band }-\left(10.25 e^{-d_{k}}\left[1-\tanh \left[6\left(\frac{h}{h_{a}}-0.625\right)\right]\right\rceil-0.33\right)
\end{aligned}
$$

Corr_band is the correction factor of the band ratio, which is equal to $0 \mathrm{~dB}$ when $B W W i M A X<B W F S S$. Otherwise, Corr_band $=-10 \log (B W W i M A X / B W F S S)$, when $B W W i M A X>B W F S S$. Therefore, when the bandwidth of FSS is $230 \mathrm{kHz}$, the correction band is given by the following expression:

$$
\text { Corr_band }=-10 l \log { }_{10}\left(\frac{20 \times 10{ }^{6}{ }_{\text {WMAM }}}{230 \times 10^{3}{ }_{\text {FSS }}}\right)=-19.4 d B
$$

The value of correction band is used in the simulation processes.

\section{Tools, Specifications and Field Measurements}

The VSAT unit used for running the field test, which receives the internet signal at 4040 MHz. A harmful interference was applied on the FSS receiver using synthesized signal generator to generate interfered signal within the range 3700-4200 MHz. The FSS unit is installed to receive an internet Bandwidth (Burstable to 256Kbps downlink and $9.6 \mathrm{kbps}$ uplink) through MEASAT III Geostationary Satellite Orbit.

The synthesized signal generator was used to generate an interference signal to assess the interference of $1 \mathrm{MHz}$ bandwidth. This is done to verify the effect of adjacent interference level as well as the in-band interference. The MEASAT 3 satellite orbit position is $91.50 \mathrm{E}$, while the dish is located at latitude of $1.5580 \mathrm{~N}$ and longitude 103.60 E Longitude. The distance of the earth station to the satellite is $35955 \mathrm{~km}$. The signal delay is $239 \mathrm{~ms}$ for MEASAT-3. For the receiver unit, antenna diameter is $1.8 \mathrm{~m}$, centre frequency $(\mathrm{Fc})$ is 4040 $\mathrm{MHz}$, elevation angle is 740 , azimuth is 263.70 , height is $1.8 \mathrm{~m}$, Bandwidth is $230 \mathrm{kHz}$ and Theoretical Interference level (I) equal to $-165 \mathrm{dBw} / 230 \mathrm{KHz}$. For the Broadband wireless access, centre frequency is $4040 \mathrm{MHz}$, peak output power is $20 \mathrm{dBm}$, channel bandwidth $1 \mathrm{MHz}$, antenna gain $10 \mathrm{dBi}$ and antenna height is $2.2 \mathrm{~m}$.

The measurements procedure with the shielding technique is started with antenna measurements, where a Horn antenna is used in the shielding experiment to represent the BWA sector. The measured return loss of the horn antenna gave a good response for the frequency band 3700-4200 MHz. An empirical experiment has been conducted using the anechoic chamber to measure the free line of sight signal level. Concurrently, a Broadband Wireless Access (BWA) signal generator is used as a WiMAX transmitter. Having set such a typical ambience, various types of metals are located in between the transmitter and the receiver. This is aimed at measuring the signal penetration through different materials in order to obtain the power loss through several barriers. The results of attenuation obtained with different shielding materials are reported in Table 1. 


\section{Table 1. Measurements of Signal Losses for $4040 \mathrm{MHz}$ Radio Paths Obstructed by Common Materials}

\begin{tabular}{|l|c|}
\hline \multicolumn{1}{|c|}{ Material type } & $\begin{array}{c}\text { Loss } \\
(\mathbf{d B})\end{array}$ \\
\hline Aluminium shield $(0.1 \mathrm{~cm}$ thickness $)$ & 22.1 \\
\hline $\begin{array}{l}\text { Aluminium mesh wire shielding }(0.2 \mathrm{~cm} \text { wire } \\
\text { spacing) }\end{array}$ & 20.9 \\
\hline Copper shielding (0.1cm thickness) & 24.5 \\
\hline $\begin{array}{l}\text { Copper mesh wire shielding }(0.2 \mathrm{~cm} \text { wire } \\
\text { spacing) }\end{array}$ & 23.3 \\
\hline Zinc shield $(0.1 \mathrm{~cm}$ thickness) & 20 \\
\hline
\end{tabular}

The losses obtained are in the range of 20 to $22.1 \mathrm{~dB}$ for the materials used as shown in Table 1. For cost-effective deployment, a zinc metal of $0.1 \mathrm{~cm}$ thickness is used to shield the FSS as shown in Figure 1.

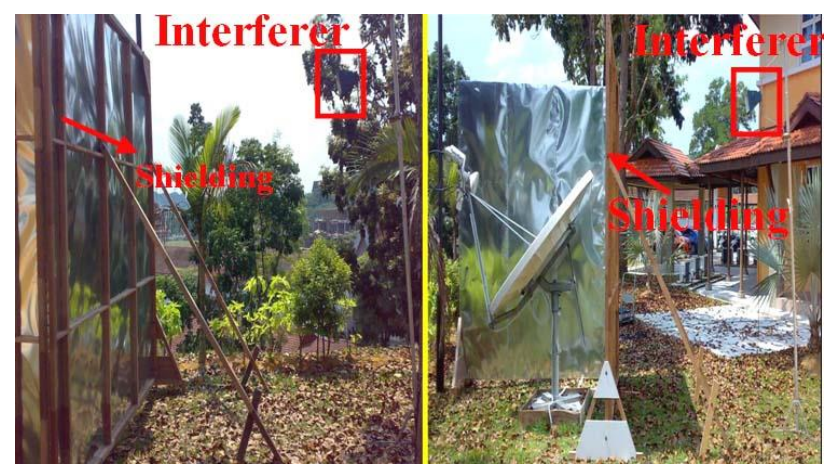

\section{Figure 1. FSS Shielding using Zinc Sheet Material}

The study has practically proved that best shielding condition occurs when the FSS receiver antenna is entirely shielded except for the top side. Furthermore, the shield should be separated at least $1 \mathrm{~m}$ from the basement of antenna and $0.5 \mathrm{~m}$ higher than the antenna's body. It must also be grounded. If a shield is deployed in the direction of the satellite, the angle of elevation from the bottom of the antenna reflector to the top of the shield should be about 5 degrees less than the satellite elevation.

A BWA synthesized signal generator is used to generate an interfering signal, which ranges from 3400 to $4200 \mathrm{MHz}$. This frequency range covers both cases of CCI and ACI as shown in Fig.1. A $20 \mathrm{dBm}$ signal with a bandwidth of $1 \mathrm{MHz}$ was generated and broadcasted in the direction of FSS receiver. The frequency of the interferer was varied from $3800 \mathrm{MHz}$ to $4100 \mathrm{MHz}$, which resulted in $0 \mathrm{~kb} / \mathrm{s}$ downlink signal in the FSS receiver. However, when the transmitter power of interferer is reduced by $1 \mathrm{dBm}$ significant decrease in interference was observed. In order to have a minimum separation distance required for the co-existence in $\mathrm{CCI}$ scenario, the deterministic calculation is given by:

$$
\begin{aligned}
& 20 \log (\mathrm{d})=\mathrm{EIRP}(-65 \mathrm{dBw})-\mathrm{I}(-165 \mathrm{dBW} / 0.23 \mathrm{MHz}) \\
& +\mathrm{Gr}(-10)-92.44-20 \operatorname{logf}(4.02 \mathrm{GHz}) ; \mathrm{d}=0.187 \mathrm{Km}
\end{aligned}
$$

Where $\mathrm{d}$ is the separation distance in $\mathrm{km}$, EIRP is the effective isotropic radiation power of the interferer, $\mathrm{I}$ is the interference level, $\mathrm{Gr}$ is the received gain, $\mathrm{f}$ is the receiving frequency of 
FSS. A $0.187 \mathrm{~km}$ is a large separation distance for a small transmitted power like $20 \mathrm{dBm}$. Therefore, the experiment has shown that co-existence scenarios based on co-channel sharing is almost practically impossible. The analyzed interfered signal collected in Table 2 was used to ensure the wave propagation attenuation after and before the FSS frequency carrier.

\section{Table 2. Effect of BWA Signal on the FSS Carrier with and Without Shielding}

\begin{tabular}{|c|c|c|c|c|c|}
\hline \multirow{2}{*}{\multicolumn{2}{|c|}{}} & \multicolumn{3}{|c|}{$20 \mathrm{dBm}$ Signal generator } \\
\cline { 3 - 6 } \multicolumn{2}{|c|}{ Parameter } & \multicolumn{2}{|c|}{$\begin{array}{c}\text { Without } \\
\text { shielding }\end{array}$} & \multicolumn{2}{c|}{$\begin{array}{c}\text { With } 0.1 \mathrm{~cm} \\
\text { Zinc }\end{array}$} \\
\cline { 2 - 6 } & $\begin{array}{c}\text { before } \\
\text { carrier }\end{array}$ & $\begin{array}{c}\text { after } \\
\text { carrier }\end{array}$ & $\begin{array}{c}\text { before } \\
\text { carrier }\end{array}$ & $\begin{array}{c}\text { after } \\
\text { carrier }\end{array}$ \\
\hline \multirow{4}{*}{$\begin{array}{c}\text { ACS } \\
\text { (dB) }\end{array}$} & $\begin{array}{c}5 \mathrm{MHz} \\
\text { Offset }\end{array}$ & 15 & 50 & 35 & 70 \\
\cline { 2 - 6 } & $\begin{array}{c}10 \\
\mathrm{MHz} \\
\text { Offset }\end{array}$ & 31 & 60 & 61 & 105 \\
\cline { 2 - 6 } & $\begin{array}{c}15 \\
\mathrm{MHz} \\
\text { Offset }\end{array}$ & 55 & 70 & 0 & 0 \\
\hline
\end{tabular}

As clearly shown in Table 2, the higher the transmission frequency, the higher is the propagation losses; and reducing the transmitted power corresponds to a reduced ability to penetrate the walls. Therefore, the effects of interference, with or without shielding, at different frequency offsets is summarized in Figure 2. The threshold value is defined at -125 $\mathrm{dBm}$.

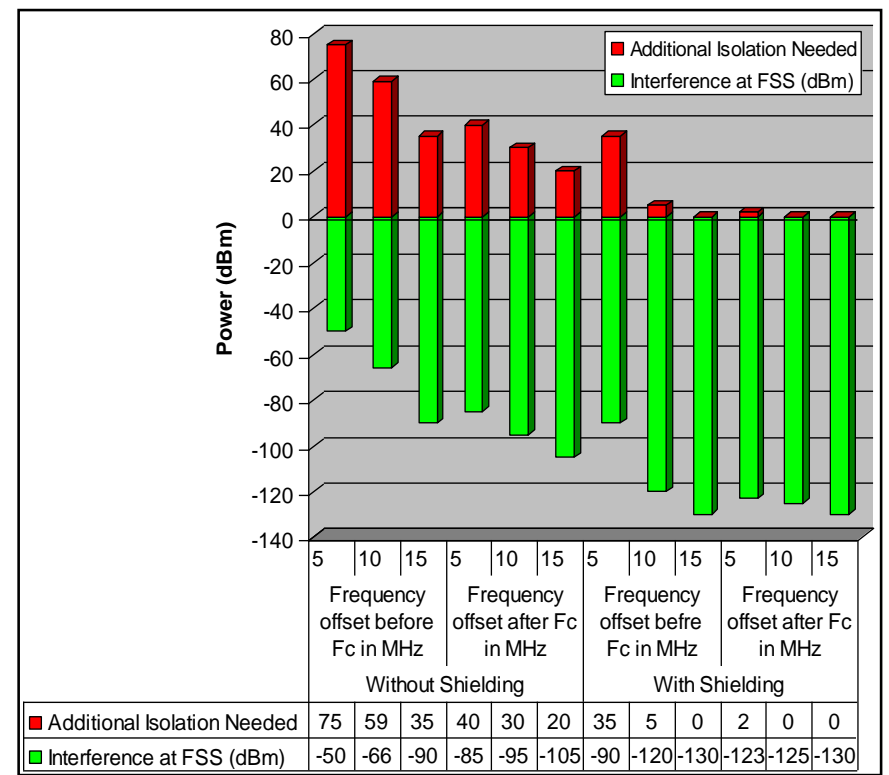

Figure 2. The Additional Isolation Needed for the FSS Protection 
Fig. 2 clearly shows that signal ability to interfere is bigger when it has less value than FSS frequency carrier and vice versa. In addition, signal attenuation is higher when shielding technique is used compared to the signal attenuation before the shielding. It is also shown in the figure that co-existence is achieved with $20 \mathrm{~dB}$ shielding attenuation and $15 \mathrm{MHz}$ frequency offset. It can be concluded that it is possible to reduce down the harmful interference to $10 \%$ by increasing the shielding attenuation to $20 \mathrm{~dB}$ and the separation distance can be reduced down to $1 \%$ for $40 \mathrm{~dB}$ shielding attenuation.

\section{The Coexistence Analysis of FSS with WiMAX using Shielding}

According to the shielding experiment at CCI scenario, the zero-guard band and guard bands separation channel are simulated to represent the interference scenarios. A minimum separation in two dimensions (frequency and distance) for different deployment areas with and without using the shielding technique has been covered.

Firstly, when the interfering signal shares the same band with the victim FSS receiver and thus separation distance is desired. Secondly, when the interfering signal is contiguous to the victim band and finally when a guard band is in between the bands in question. The worst case of sharing between WiMAX and FSS receiver is simulated when both the interfering and victim antennas are opposite-tower-mounted and facing each other. An FSS antenna of variable heights $(1.8 \mathrm{~m}$ and $5 \mathrm{~m})$ has been used to emphasize that positioning the FSS receiver onto the ground can effectively reduce the separation.

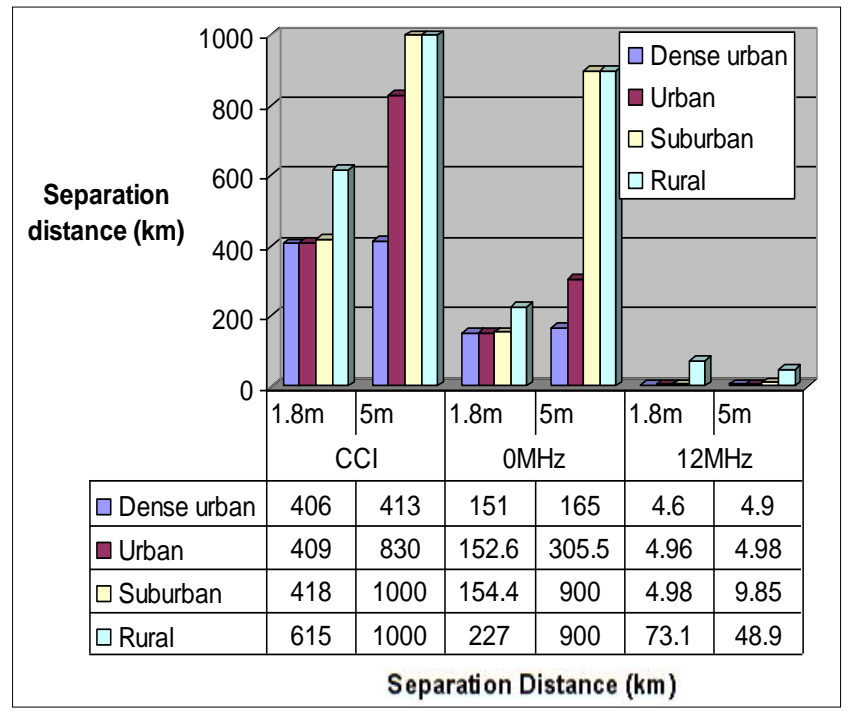

Figure 3. The Separation Distance between WiMAX and FSS when FSS Bandwidth is $0.23 \mathrm{MHz}$ for Four Deployment Areas for $\mathrm{CCl}$, Zero Guard Band and $12 \mathrm{MHz}$ Guard Band with $20 \mathrm{~dB}$ Shielding Attenuation

In Fig.3, the $20 \mathrm{~dB}$ shielding mitigation technique is used to reduce the separation distance between the two services, which corresponds to the reduction of $10 \%$ of the original distance. The reduced separation obtained in Fig.3 (with the insertion of $12 \mathrm{MHz}$ guard band and $20 \mathrm{~dB}$ Shielding) is not sufficient for practical deployment of the future communications systems. Obviously, a minimum separation distance is calculated for $36 \mathrm{MHz}$ FSS bandwidth when shielding attenuations are 0 and $20 \mathrm{~dB}$, respectively. Figure 4 shows the results for $36 \mathrm{MHz}$ 
FSS $(1.8 \mathrm{~m}$ ) bandwidth when $\Delta \mathrm{f}=0$ (CCI), 28 (zero guard band), 33 (12 MHz guard band) and $40 \mathrm{MHz}$ for the four deployment areas.

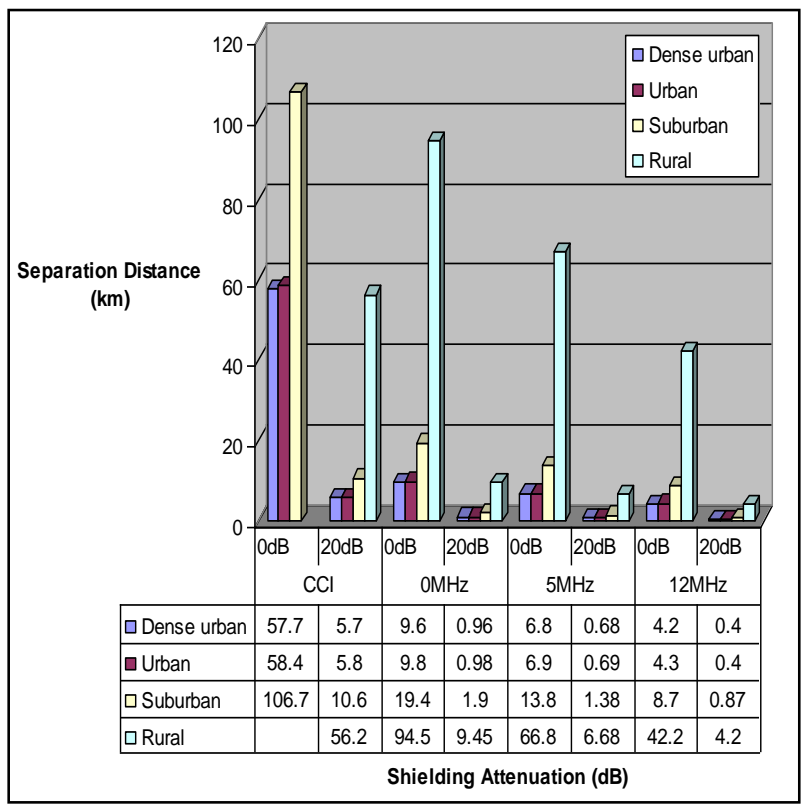

Figure 4. The Separation Distance between WiMAX and FSS when FSS Bandwidth is $36 \mathrm{MHz}$ in the Four Deployment Areas for $\mathrm{CCl}$, Zero Guard Band and $12 \mathrm{MHz}$ Guard Band and $40 \mathrm{MHz}$ with 0 and $20 \mathrm{~dB}$ Shielding Attenuation

From Fig.4, it is noticed that separation distance reduced to $0.4 \mathrm{~km}$ when $12 \mathrm{MHz}$ is used as guard band (with a $20 \mathrm{~dB}$ as a shielding attenuation in dense urban area deployment). However co-existence in the CCI scenario is still difficult due to large separation distance required. Since base station-to-base station is the main scenario of interference, complete analyses on the antenna discrimination effect should be done by using the smart antenna, being a suggested technology for next generation of mobile communication.

\section{Antenna Discrimination Impacts}

An Antenna Discrimination Loss (ADL) is the difference in azimuth between the interferer antenna direction and the victim receiving direction [17]. Thus, pointing the beams of antenna victim and interferer are not aligned on each other, and it could lead to degradation in the interferer gain toward the victim. In order to highlight this issue, the separation distance results obtained in Fig.5 for a dense urban area deployment is incorporated in the ADL simulation. Figure 5 shows the varying values of minimum separation distance using ADL in the range of 0 to $15 \mathrm{~dB}$ of the CCI, with 0 and $12 \mathrm{MHz}$ guard band separation. Definitely, the ADL technique proposes another mitigation technique which supports the smart antenna technology. However, a scenario of intersystem interference is also considered in order to compare the effect of three sectored terrestrial base station and electrically shifted beam base station on the FSS earth station [18]. 


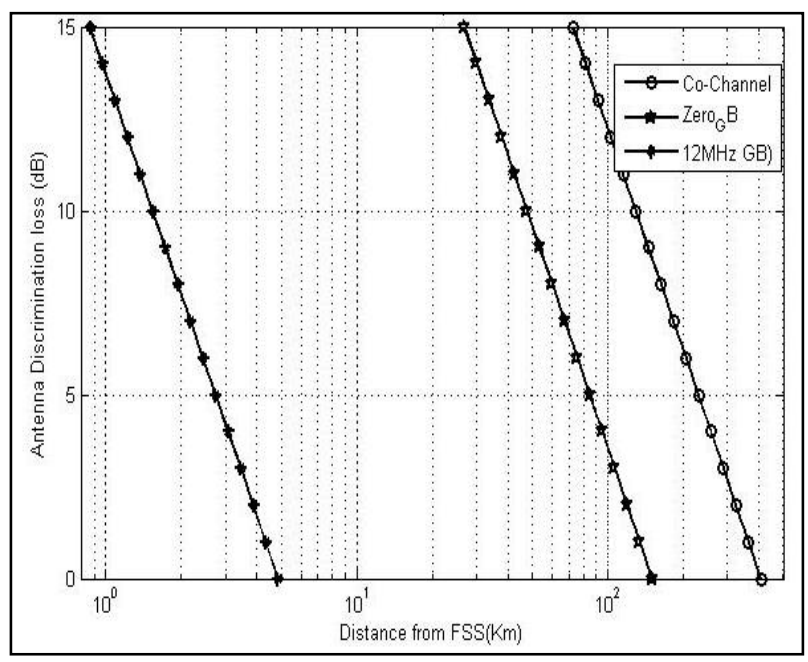

\section{Figure 5. Antenna Discrimination Loss and Minimum Separation Distance when FSS Bandwidth is $0.23 \mathrm{MHz}$ in Dense Urban Area with $20 \mathrm{~dB}$ Shielding Attenuation}

In the cases of co-channel co-existence, zero guard band and adjacent channel, a $15 \mathrm{~dB}$ antenna discrimination loss can decrease the physical separation from $406 \mathrm{~km}, 165 \mathrm{~km}$ and 49 $\mathrm{km}$ to $72 \mathrm{~km}, 26.8 \mathrm{~km}$ and $0.86 \mathrm{~km}$, respectively, for $20 \mathrm{MHz}$ WiMAX channel bandwidth and $1.8 \mathrm{~m}$ FSS height with $20 \mathrm{~dB}$ shielding protection in dense urban area.

\section{Conclusion}

The proposed shielding technique proves resilient in the presence of interference (with or without the guard band). The technique may thus be considered as a viable alternative to many other commercial-of-the-shelf (COTS) mitigation solutions. On the shielding mitigation technique, it was found that different materials have different levels of signal attenuation. The proposed shielding material $(0.1 \mathrm{~mm}$ thickness Zinc sheet) was a balanced choice, providing a high attenuation (about $20 \mathrm{~dB}$ ) at a lower cost, compared to other metals. It was also found that it is possible to reduce the harmful interference up to $10 \%$ by increasing the shielding attenuation up to $20 \mathrm{~dB}$. Consequently the separation distance can be minimized to $1 \%$ for 40 $\mathrm{dB}$ shielding attenuation. This method can be applied to other satellite systems, because different channel bandwidths were simulated for the victim FSS receiver. The simulation results have shown that both the interference and separation distance decrease with an increasing channel bandwidth.

Co-channel interference scenario in the rural area is the most difficult compared to other scenarios. However, it requires a long coordination distance in the range of $6150 \mathrm{~km}$ and 86 $\mathrm{km}$ without shielding effect for a $0.23 \mathrm{MHz}$ and $36 \mathrm{MHz}$ FSS channel bandwidths, respectively, given an FSS antenna height of $1.8 \mathrm{~m}$. By adding $40 \mathrm{~dB}$ shielding attenuation, the coordination distance will correspondingly be reduced to $61 \mathrm{~km}$ and $0.86 \mathrm{~km}$. These are the highest reduction that could be achieved without guard separation. These findings emphasize that the shielding technique can significantly improve the FSS immunity against the interference as well as the signal reception via FSS. However, adjacent channel interference scenario with frequency offsets from the carrier of $12 \mathrm{MHz}$ in dense urban area shows the best co-existence scenario with $40 \mathrm{~dB}$ shielding attenuation. For instance, it needs $0.49 \mathrm{~km}$ and $0.04 \mathrm{~km}$ geographical separation for $0.23 \mathrm{MHz}$ and $36 \mathrm{MHz}$ FSS channel 
bandwidths, respectively, when FSS antenna height is $1.8 \mathrm{~m}$. This indicates that the dense urban area is the best area for co-existence and intersystem interference coordination.

From the deployment standpoint, different areas are considered and it is shown that the dense urban type of environment is the most convenient type for successful co-existence scenarios, whereas the rural one is the worst for frequency sharing and coordination in the same band. From the shielding perspective, it is worth mentioning that this technique is applicable to any antenna size at various heights. For the ADL, it is concluded that other mitigation techniques should be researched to enhance the co-existence between the two services by reducing the separation distance.

\section{References}

[1] ITU-R Document 8F/1015-E, "Sharing studies between FSS and IMT-Advanced systems in the 3400-4200 and 4500-4800 MHz bands", (2006).

[2] ITU-R WP 8F/TEMP 432 rev.2., "Working document towards a PND report on sharing studies between IMT-ADVANCED and the Fixed Satellite Service in the 3 400- 4200 and 4 500-4 $800 \mathrm{MHz}$ bands", ITU-R Working Party 8F, (2006).

[3] L. F. Abdulrazak, Z. A. Shamsan and T. A. Rahman, "Potential Penalty Distance between FSS Receiver and FWA for Malaysia", International Journal Publication in WSEAS Transactions on COMMUNICATIONS, vol. 7, no. 6, (2008), pp. 637-646.

[4] H.-S. Jo, H.-G. Yoon, J.W. Lim and J.-G. Yook, "An Advanced MCL Method for Assessing Interference Potential of OFDM-Based Systems beyond 3G with Dynamic Power Allocation", Proceedings of the 9th European Conference on Wireless Technology, (2006), pp. 39-42.

[5] P. A. Seidenberg, M.P. Schulz, E. Herbster, G. Kottkamp, "Statistical of Minimum Coupling Loss in UMTS/IMT-2000 Reference Scenarios”, Vehicular Technology Conference VTC'99, IEEE VTS $50^{\text {th }}$, vol. 2, (1999), pp: 963 - 967.

[6] ITU-R WP8F Contribution, "Proposed MIMO Channel Model Parameters for Evaluation of Air Interface Proposals for IMT-Advanced", Meeting in Cameroon, (2007).

[7] X. Li and L. J. Cimini Jr., "Effects of clipping and filtering on the performance of OFDM", IEEE Commun. Letters, vol. 2, (1998), pp. 131-133.

[8] WiMAX Forum (white paper), "Compatibility of Services using WIMAX Technology with Satellite Services in the $2.3-2.7 \mathrm{GHz}$ and $3.3-3.8 \mathrm{GHz}$ Bands", (2007).

[9] ITU-R WP 8F/975-E, "Analysis of interference from IMT to FSS in the 3400-4200 MHz and 4500-4800 MHz", (2006).

[10] RSAC Paper, "Assessment of Potential Interference between Broadband Wireless Access Systems in the 3.4 - 3.6 GHz Band and Fixed Satellite Services in the 3.4 - 4.2 GHz Band", (2006).

[11] Radiocommunication Study group, "Update of Sharing Studies between IMT-Advanced and Fixed Satellite Service in the 3 400-4 200 and 4 500-4 800 MHz Bands", Document 8F/927-E, (2006).

[12] ITU-R SF.1486, "Sharing methodology between Fixed Wireless Access Systems in the Fixed Service and Very Small Aperture Terminals in the Fixed-Satellite Service in the 3 400-3 $700 \mathrm{MHz}$ Band", ITU-R R WP4-9S, (2000).

[13] ITU-R Recommendation SF.1006, "Determination of the interference potential between earth stations of the fixed-satellite service and stations in the fixed service, (1993).

[14] X. Li and L. J. Cimini Jr., "Effects of clipping and filtering on the performance of OFDM", IEEE Commun. Letters, vol. 2, (1998), pp. 131-133.

[15] Recommendation ITU-R P.452-12, "Prediction Procedure for the Evaluation of Microwave Interference between Stations on the Surface of the Earth at Frequencies above about $0.7 \mathrm{GHz}$ ", (2007).

[16] Document AWF-3/17, "Assessment of potential interference between Broadband Wireless Access (BWA) in 3.4-3.6 GHz band and Fixed Satellite Service (FSS) in 3.4-4.2 GHz band", Office of the Telecommunications Authority (OFTA) Hong Kong, (2006).

[17] Z. A. Shamasn, L. Faisal and T. A. Rahman, "Coexistence and Spectrum Sharing between IMT-Advanced and Existing Fixed Systems", International Journal Publication in WSEAS TRANSACTIONS on COMMUNICATIONS, volume 7, no. 5, (2008), pp. 505-515.

[18] Z. A. Shamasn, L. Faisal, S. K. Syed-Yusof, T. A. Rahman, "Spectrum Emission Mask for Coexistence between Future WiMAX and Existing Fixed Wireless Access Systems", International Journal Publication in WSEAS Transactions on COMMUNICATIONS, volume 7, no. 6, (2008), pp. 627-636. 